\title{
Breast tuberculosis: Rare but still present in Italy - A case of mycobacterium breast infection
}

\author{
M.L. Meggiorini ${ }^{\mathrm{a}, *}$, D. Vitolo ${ }^{\mathrm{b}}$, A. Russo $^{\mathrm{a}}$, V. Trinchieri ${ }^{\mathrm{c}}$ and C. De Felice ${ }^{\mathrm{d}}$ \\ ${ }^{a}$ Department of Obstetric-Gynecological and Urological Sciences, University of Rome “Sapienza”, Rome, Italy \\ ${ }^{\mathrm{b}}$ Division of Oncology, Department of Radiological, Oncological and Pathological Sciences, Sapienza University, \\ Rome, Italy \\ cDepartment of Public Health and Infectious Diseases, University of Rome "Sapienza", Rome, Italy \\ ${ }^{\mathrm{d}}$ Department of Radiological Sciences, University of Rome "Sapienza”, Rome, Italy
}

\begin{abstract}
Breast tuberculosis is a rare disease in highly endemic countries, and it is even rarer in Western countries, where only occasionally the local population is affected. The rarity of the disease and particularly the lack of a typical clinical-radiological presentation may cause tuberculosis to be mistaken for breast cancer or a pyogenic abscess. The authors present a case of breast tuberculosis in a 27-year-old nulliparous woman, an Italian citizen of the Caucasian race, who has never resided in a tuberculosis endemic area. She presented with painful retroareolar and para-areolar swelling in the right breast associated with cutaneous hyperemia (without fistulization), resistant to antibiotic therapy. Histopathological examination revealed features of mastitis with epithelioid histiocytes and Langhans giant cells and was characterized by the presence of caseous necrosis which suggested tuberculous inflammation. Ziehl-Neelsen staining showed the presence of acid fast bacilli. In countries where tuberculosis is non-endemic, breast tuberculosis should always be included in the differential diagnosis in cases of inflammatory breast lesions resistant to the usual antibiotic therapies. Early recognition may prevent both clinical progression and surgical excision, as breast tuberculosis usually regresses as a response to appropriate anti-tuberculosis therapy.
\end{abstract}

Keywords: Breast tuberculosis, Caucasian Italian woman

\section{Introduction}

Tuberculosis infection commonly affects the lungs. However, in $17.9 \%$ of cases it affects extrapulmonary organs and tissues [1]. The first case of breast tuberculosis was described in 1829 by Cooper, who defined it as a "scrofulous swelling of the bosom" [2]. The breast is a rare site of extrapulmonary mycobacterial infection and it accounts for only $0.1 \%-0.5 \%$ of all cases of tuberculosis [3]. Even in tuberculosis endemic countries,

\footnotetext{
*Corresponding author: Maria L. Meggiorini, Department of Obstetric-Gynecological and Urological Sciences, University of Rome "Sapienza", Policlinico Umberto I, Viale del Policlinico 155, 00161 Rome, Italy. Tel.: +39 3470385597; Fax: +39 06499772317; E-mail: marialetizia.meggiorini@uniroma1.it.
}

breast tuberculosis is rare. In India the incidence of tuberculosis is 185 per 100,000 Population [4]. According to the literature the incidence of breast tuberculosis varies from $0.1 \%$ to $3 \%$ of surgically treated breast diseases [5]. In Western countries the reported incidence is less than $0.1 \%$ of all breast specimens submitted to histological examination [6]. The highest incidence occurs in multiparous women of childbearing age during lactation or pregnancy or associated with injury or trauma to the breast $[7,8]$.

Both breasts are equally affected [9] but bilateral synchronous infection occurs only in $3 \%$ of cases [10].

McKeown and Wilkinson distinguished between two types of breast tuberculosis: primary and secondary infection [11]. Primary infection is extremely rare and is caused by ductal infection in response to abrasion or 
external opening of the nipple ducts [12]. Contagion from breastfed infants affected by tuberculosis cannot be excluded [9]. Secondary infection is a result of dissemination of a primary infection. The most common spread is by continuity and contiguity through the bloodstream and lymphatic vessels [8,11]. Progressive tuberculous infection of the breast passes from the tracheobronchial lymph nodes to the tracheal lymph nodes and to the mediastinal and internal breast lymph nodes [11].

Breast tuberculosis has no well-defined clinical and radiological features [9] and the lesion is therefore often mistaken for carcinoma or pyogenic abscess [1315]. Only fine needle aspiration cytology (FNAC) or histological examination can provide a definitive diagnosis $[6,16,17]$. Treatment with anti-tuberculosis drugs is an essential part of therapeutic management which may or may not include surgery $[7,18,19]$.

\section{Case report}

In October 2010, a 27-year-old woman, an Italian citizen of the Caucasian race, was referred to the Policlinico Umberto I University Hospital in Rome due to a painful swelling of the right breast. It had appeared about 10 days earlier and was associated with redness of the skin. Clinical signs suggested retroareolar abscess.

Informed consent was obtained from the patient for the publication of this case report.

The patient had undergone breast duct excision of the left breast due to a similar retroareolar lesion in 2007 , and the histological report described multiple foci of chronic granulomatous mastitis. The patient had no family history of breast cancer; she was nulliparous and not pregnant. There was no complaint of fever, weight loss or night sweats, no fatigue and no history of tuberculosis. She was a nonsmoker and reported no drug and/or alcohol use. She lived in a rural area, but not in contact with animals. She had not had any unpasteurized milk or dairy products. She had hired a cleaning lady of Indian origin in 2008-2009 and had been to Sharm el Sheik for a period of 2 weeks in April 2010. In Egypt the incidence of tuberculosis is 18 per 100,000 Population, much lower than the incidence in endemic countries [20].

Physical examination revealed a painful retroareolar and para-areolar swelling in the right breast associated with cutaneous hyperemia without fistulization. The ipsilateral axillary lymph nodes were not palpable.
Ultrasound (US) examination of the breast showed a hypoechoic irregular area of about $14 \times 8 \mathrm{~mm}$ with ill defined margins. FNAC of the mass was recommended but not performed as the patient refused. Antibiotic therapy was prescribed $(875 \mathrm{mg}$ amoxicillin and $125 \mathrm{mg}$ clavulanic acid), 1 tablet every 12 hours for 7 days resulting in a modest reduction in the swelling.

Due to persistence of symptoms, the patient requested surgical excision of the swelling in view of her previous medical history of contralateral breast inflammation which was satisfactorily resolved with surgical therapy. However, the patient had several work commitments, and surgery was therefore delayed for two months. In this period, the clinical symptoms worsened with onset of fistulization, and the patient lost about $5 \mathrm{~kg}$. However, she put the weight-loss down to a diet initiated in the same period.

Before surgery blood tests were performed; all parameters were normal. Conventional chest $\mathrm{x}$-ray in two projections revealed no focal pathology. In January 2011 excisional biopsy was performed. Histopathological examination of the surgical specimen revealed the presence of mastitis with epithelioid histiocytes, Langhans giant cells and the presence of caseous necrosis (Fig. 1). Ziehl-Neelsen staining showed the presence of acid fast bacilli (Fig. 2). A specialist in infectious diseases was therefore consulted, and at the same time a rereading of the previous histology slides was requested.

Further investigation included the Mantoux tuberculin skin test, QuantiFERON-TB Gold and search for acid fast bacilli in the urine, but all tests yielded negative results. A comprehensive study of cell-mediated immunity was also carried out but revealed no type of immune deficiency.

In this case, bacterial culture was not possible. After surgical excision of the lesion, the entire specimen was submitted to histological examination and no material was available for further analysis.

The patient was administered oral anti-tuberculosis therapy: rifampicin $600 \mathrm{mg} /$ day, isoniazid $300 \mathrm{mg} / \mathrm{day}$, ethambutol $1200 \mathrm{mg} /$ day and pyrazinamide $1500 \mathrm{mg} /$ day for 4 months, followed by an additional 2-month therapy of rifampicin $600 \mathrm{mg} /$ day and isoniazid $300 \mathrm{mg} /$ day. After completed treatment, physical examination and US scanning showed that the lesion had disappeared.

Histological rereading of the initial specimen (2007) showed granulomatous mastitis with epithelioid histiocytes and Langhans giant cells; the result of ZiehlNeelsen staining was negative. 


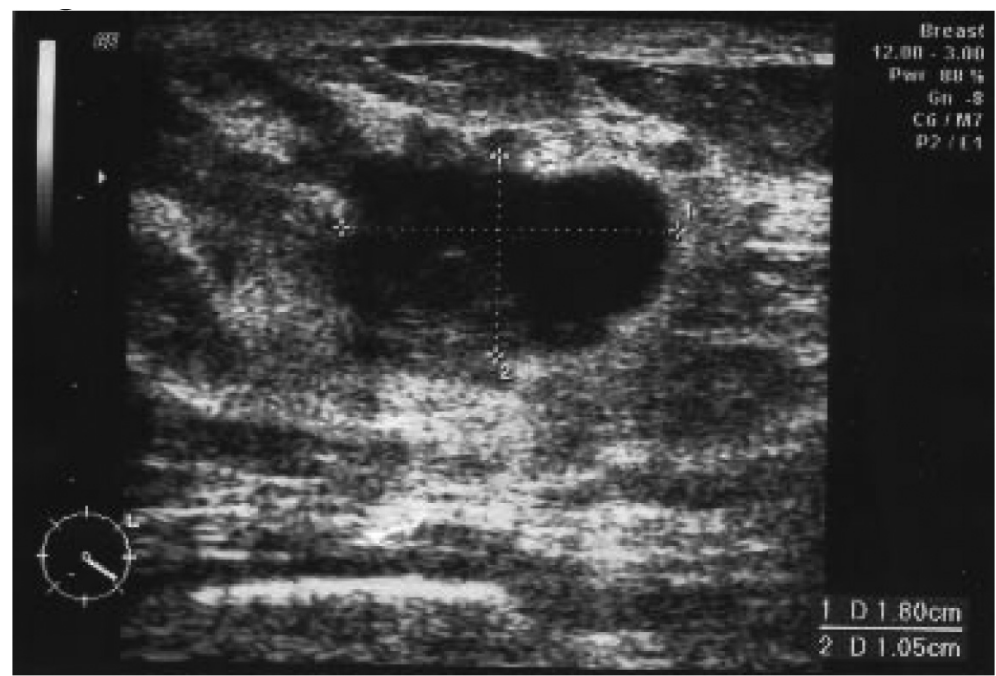

Fig. 1. A complex hypo/anechoic nodule containing fluid and solid parts; margins partly illdefined; the nodule is adjacent to one of the main milk ducts.

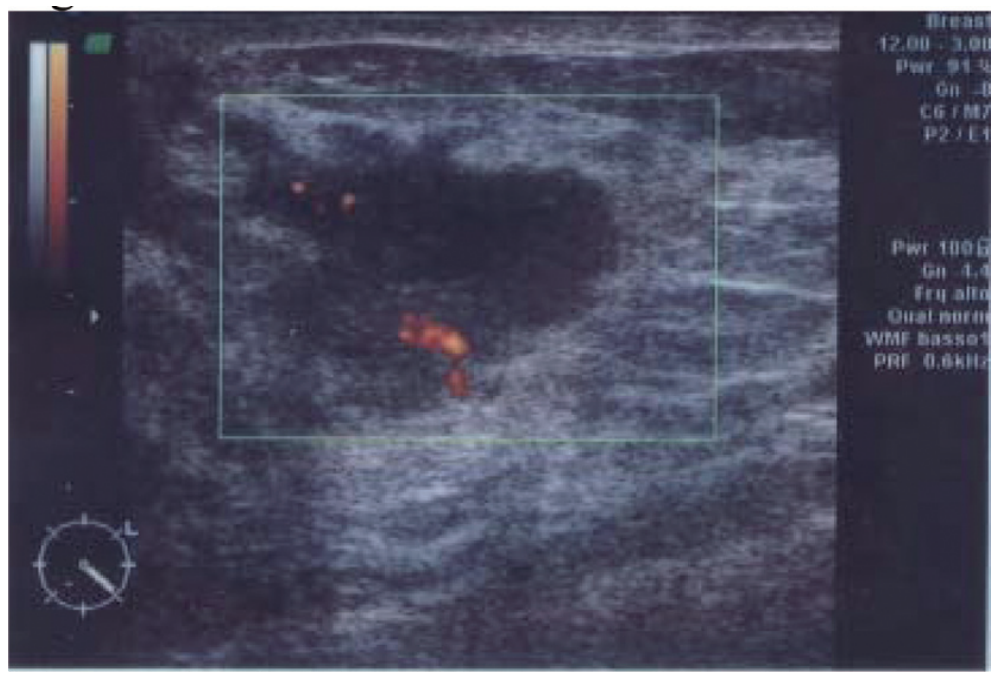

Fig. 2. Color Doppler shows blood flow signals in the peripheral solid component. (Colours are visible in the online version of the article; http://dx.doi.org/10.3233/BD-2012-000338)

\section{Discussion}

Breast tuberculosis (TB) is an extremely uncommon disease even in endemic areas and it is even rarer in the Western countries [1,21], where only occasionally the local population is affected [22].

The rarity of the disease and particularly the lack of a typical clinical-radiological presentation [9] can cause tuberculosis to be mistaken for breast cancer or a pyogenic abscess [14-16]. Clinical examination often fails to differentiate breast carcinoma from tuberculosis. A high index of suspicion is therefore necessary to make a correct diagnosis. Factors predictive but not diagnostic of breast tuberculosis include mobile breast lump, multiple sinuses as well as constitutional symptoms [10] in young, multiparous or lactating females [7]. In contrast, nipple retraction, peau d'orange appearance, and involvement of axillary lymph nodes are more common in breast cancer [18].

Modern radiological techniques can detect the presence of a lesion and assess the extension, but they do not allow a differential diagnosis of breast cancer versus mastitis [23] such as idiopathic granulomatous mastitis, mycotic mastitis, sarcoidosis, Wegener's granulo- 


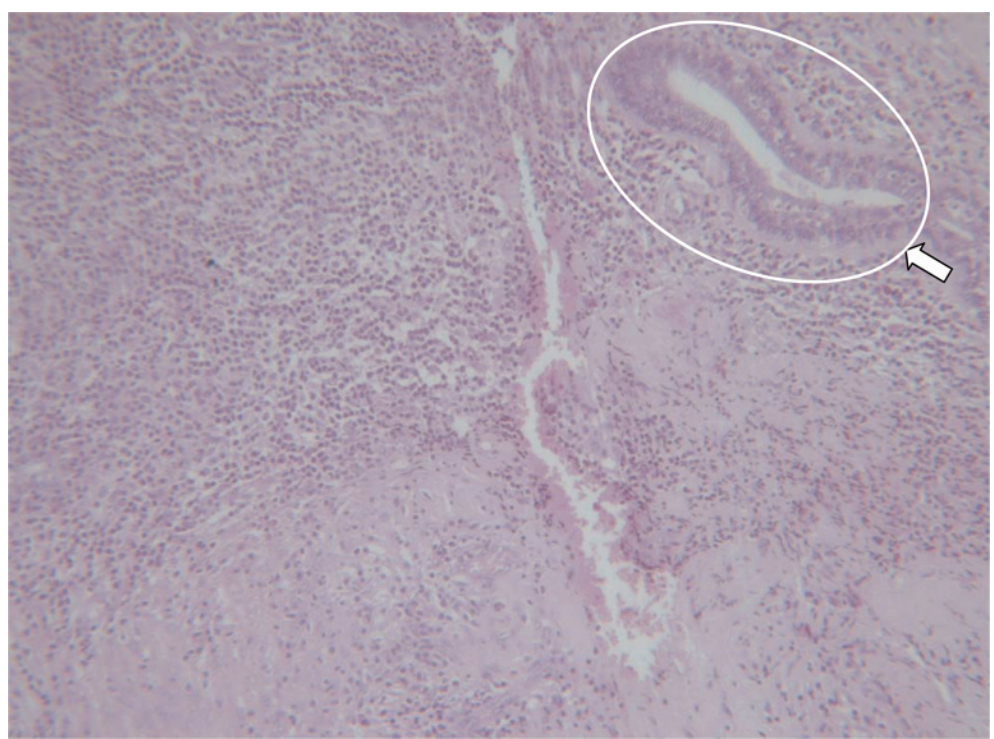

Fig. 3. Granulomatous mastitis: In the upper-right side of the picture there are remnants of a lobular duct, whereas the acinar structures are completely effaced by the granulomatous inflammation. Hemat/Eosin x 125. (Colours are visible in the online version of the article; http://dx.doi. org/10.3233/BD-2012-000338)

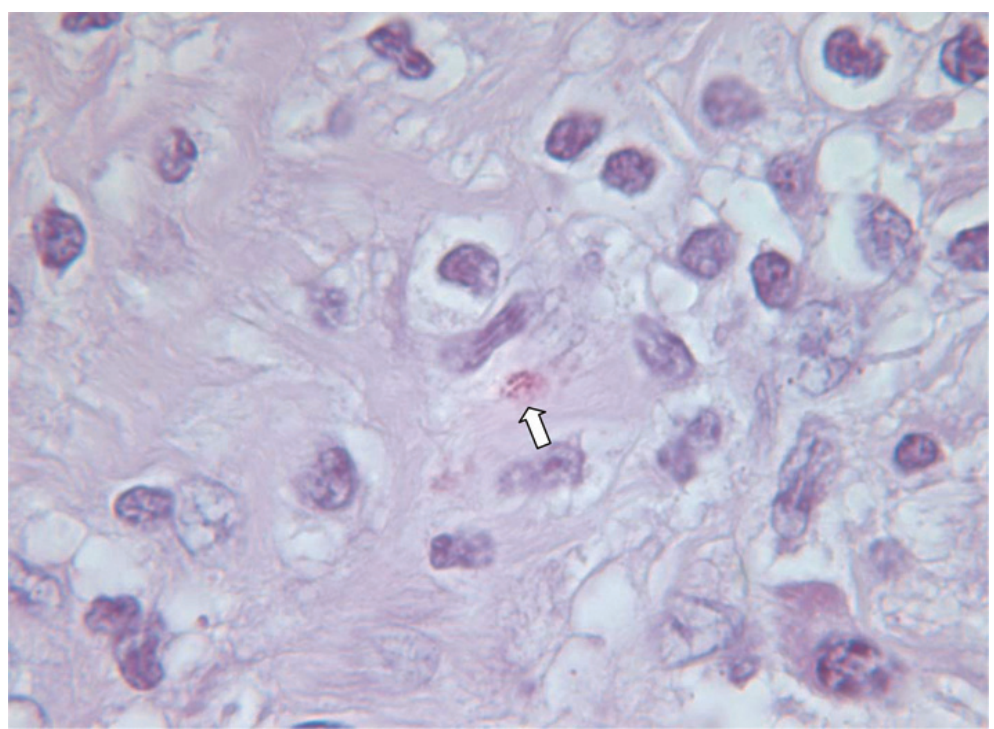

Fig. 4. Ziehl-Neelsen staining allows to detect intracellular bacteria. Counterstained with hematoxylin $\mathrm{x} 400$. (Colours are visible in the online version of the article; http://dx.doi.org/10.3233/BD-2012-000338)

matosis, pyogenic abscess, and giant cell arteritis [23, 24].

The peculiarity of the present case is the fact that the patient is an Italian woman under 30; she lives in Italy and has never resided in a tuberculosis endemic area. At the time of presentation to our department in October 2010 the clinical picture was suggestive of nonspecific inflammation of the breast. US examination was therefore performed revealing a hypoechoic area. US image was compatible with abscess but this technique did not permit exclusion of tumor. The patient refused to undergo FNAC and she was therefore administered empirical treatment with amoxicillin-clavulanic acid as it was hypothesized that she had a breast abscess caused by bacteria. As the clinical picture persisted after antibiotic therapy, the patient was submitted to excisional 
biopsy of the mass located in the right breast, and subsequent histological examination provided diagnosis of breast tuberculosis because of the histological pattern and the presence of acid fast bacilli (Fig. 2).

The following factors contributed to the incorrect preoperative diagnosis: a) the rarity of the disease in Caucasian women living in an area non-endemic for tuberculosis [21]; b) the failure to perform a direct microscopic examination and culture of the abscess material excised in 2007 ; c) the patient's refusal to undergo FNAC, which in $70 \%-75 \%$ of cases provides a diagnosis of breast tuberculosis due to the presence of Langhans cells, lymphoid aggregates and granulomatous inflammation [16].

It should be kept in mind that acid fast bacilli are found only in $25 \%$ of specimens as detection requires a concentration of 10,000-100,000/ml [25]. Definitive diagnosis of breast tuberculosis is generally made after excisional biopsy and histological examination evidencing the presence of Langhans giant cells and caseous necrosis, which is pathognomonic of tuberculosis $[6,16,17]$.

In the described case it can be established that the second lesion excised in 2011 was caused by tuberculosis in view of the histological outcome and the presence of acid fast bacilli. Most likely it was a primary lesion, as chest X-ray and search for acid fast bacilli in the urine were all negative. The fact that the Mantoux tuberculin skin test and the QuantiFERON-TB Gold were negative could be explained either by the failure of the patient's immune system to respond or by the presence of atypical mycobacteria. As the patient was a healthy woman with an uncompromised immune system we favor the second hypothesis.

There was no evidence showing that the lungs were involved, and it is therefore likely that weight loss was caused by the diet and not by the infection.

All these data are suggestive of a local infection limited to the right breast without any signs and symptoms of systemic dissemination.

With regard to the first lesion which had occurred five years earlier, it cannot be established with certainty whether it was caused by tuberculosis. However, had the second lesion been a clinical relapse in the contralateral breast, the disease would have been disseminated through the bloodstream or lymphatic vessels. This implies that the disease would have affected other organs, but this had not happened.

The following conclusions can be drawn from this case study: a) Breast tuberculosis should always be suspected in case of inflammatory breast lesions resistant to the usual antibiotic therapies, even in countries where tuberculosis is non-endemic;

b) Breast tuberculosis should always be included in the differential diagnosis of granulomatous mastitis.

In case of inflammatory breast lesions, FNAC should always be performed and include bacterioscopic examination as well as culture of the abscess material. However, in this case, bacterial culture was not possible. After surgical excision of the lesion in 2007, the entire specimen was submitted to histological examination and no material was available for further analysis.

A careful evaluation is essential for a correct histological diagnosis, particularly in the absence of acid fast bacilli.

Early recognition may prevent both clinical progression and surgical excision, as breast tuberculosis usually regresses as a response to appropriate antituberculosis therapy. However, also when diagnosis is achieved post-surgically after excision of the lesion, as it occurred in the present case, anti-tuberculosis therapy should always be administered in the same doses and combination as administered in pulmonary tuberculosis.

\section{References}

[1] Cowie RL, Sharpe JW. Extra-pulmonary tuberculosis: a high frequency in the absence of HIV infection. International journal of tuberculosis and lung disease. 1997;1(2):159-62.

[2] Cooper A. Illustrations of the diseases of the breast. Part I. London: Longman, Rees, Orme, Brown and Green; 1829 p.73.

[3] Thompson KS, Donzelli J, Jensen J, Pachucki C, Eng AM, Reyes CV. Breast and cutaneous mycobacteriosis: diagnosis by fine-needle aspiration biopsy. Diagnostic cytopathology. 1997; 17(1):45-9.

[4] https://extranet.who.int/sree/Reports?op=Replet\&name $=\% 2$ FWHO_HQ_Reports\%2FG2\%2FPROD\%2FEXT\%2FTB CountryProfile\&ISO2=IN\&outtype $=$ html.

[5] Hamit HF, Ragsdale TH. Breast tuberculosis. J R Soc Med. 1982;75(10):764-5.

[6] Al-Marri MR, Almosleh A, Almoslmani Y. Primary tuberculosis of the breast in Qatar: ten year experience and review of the literature. Eur J Surg. 2000;166(9):687-90.

[7] Shinde SR, Chandawarkar RY, Deshmukh SP. Tuberculosis of the breast masquerading as carcinoma: a study of 100 patients. World J Surg. 1995;19(3):379-81.

[8] Mukerjee P, George M, Maheshwari HB, Rao CP. Tuberculosis of the breast. J Indian Med Assoc. 1974;62(12):410-2.

[9] Wilson TS, Macgregor JW. The diagnosis and treatment of tuberculosis of the breast. Can Med Assoc J. 1963;89:1118-24. 
[10] Banerjee SN, Ananthakrishnan N, Mehta RB, Parkash S. Tuberculous mastitis: a continuing problem. World J Surg. 1987; Feb;11(1):105-9.

[11] Mckeown KC, Wilkinson KW. Tuberculous diseases of the breast. Br J Surg. 1952;39(157):420.

[12] Goksoy E, Duren M, Durgun V, Uygun N. Tuberculosis of the breast. Eur J Surg. 1995;161(7):471-3.

[13] Sakr AA, Fawzy RK, Fadaly G, Baky MA. Mammographic and sonographic features of tuberculous mastitis. Eur J Radiol. 2004;51(1):54-60.

[14] Morino GF, Rizzardi G, Gobbi F, Baldan M. Breast tuberculosis mimicking other diseases. Tropical Doctor. 2007; 37(3):177-178

[15] Makanjuola D, Murshid K, Al Sulaimani S, Al Saleh M. Mammographic features of breast tuberculosis: the skin bulge and sinus tract sign. Clin Radiol 1996; 51: 354-358.

[16] Kakkar S, Kapila K, Singh MK, Verma K. Tuberculosis of the breast. A cytomorphologic study. Acta Cytol. 2000;44(3):2926.

[17] Sharma PK, Babel AL, Yadav SS. Tuberculosis of breast (study of 7 cases). J Postgrad Med. 1991;37(1):24-6.

[18] Tewari M, Shukla HS. Breast tuberculosis: diagnosis, clinical features \& management. Indian J Med Res. 2005;122(2):10310 .
[19] Chowdri NA, Parray FQ, Dar RA, Malik AA, Mushtaque M, Malik RA. Tubercular mastitis - a rare presentation. Int J Surg. 2010;8(5):398-400.

[20] https://extranet.who.int/sree/Reports?op=Replet\&name $=\% 2$ FWHO_HQ_Reports\%2FG2\%2FPROD\%2FEXT\%2FTB CountryProfile\&ISO2=EG\&outtype $=$ html.

[21] Akçay MN, Sağlam L, Polat P, Erdoğan F, Albayrak Y, Povoski SP. Breast tuberculosis - importance of recognition and differentiation from that of a breast malignancy: report of three cases and review of the literature. World Journal of Surgical Oncology. 2007;5:67.

[22] Green RM, Ormerod LP. Breast tuberculosis: rare but still present in the United Kingdom. Int J Tuberc Lung Dis. 2000; 4(8):788-90.

[23] Al-Marri MR, Aref E, Omar AJ. Mammography features of isolated tuberculous mastitis. Saudi Med J. 2005;26(4):64650.

[24] Sen M, Gorpelioglu C, Bozer M. Isolated primary breast tuberculosis: report of three cases and review of the literature. Clinics. 2009;64(6):607-10.

[25] Gupta D, Rajwanshi A, Gupta SK, Nijhawan R, Saran RK, Singh R. Fine needle aspiration cytology in the diagnosis of tuberculous mastitis. Acta Cytol 1999;43(2):191-4. 